\title{
Anosognosia: How to Treat the Lack of Insight in Mental Health
}

\author{
Henry $\mathrm{AM}^{*}$ \\ Department of Mental Health and Substance Dependence, Alquimedez Mental Health \\ Counseling, USA
}

*Corresponding author: Henry A Montero, Director of Behavioral Health and Care

Management, Alquimedez Mental Health Counseling, PO Box 230 Yonkers, NY 10702, USA, Email: henry@alquimedez.com

\begin{tabular}{|c|}
\hline Case Report \\
Volume 3 Issue 5 \\
Received Date: June 01, 2018 \\
Published Date: June 28, 2018 \\
\hline
\end{tabular}

\section{Abstract}

Even though the scope of mental issues is relatively broad, Anosognosia is a critical psychological issue characterized by lack of self-awareness on the existence of a medical condition. In this condition, the patient lacks the rational capacity to comprehend the essence of medical attention. Notably, the existence of similar scenario is not voluntary as the neuron in the front part of the brain has been affected. The essence of the paper analyses the case of Ann W., a patient who has had symptoms that are disposed towards Anosognosia. Through the evaluation, the researcher depicts the vast range of characteristics that define this medical condition. Significantly, this condition is more prevalent among individuals who have demonstrated adverse mental disorders, such as bipolar disorder, and stroke. The most notable approach that can be used in diagnosis is the demonstration of adverse neglect of a person to seek medical assistance even when faced with precarious health situations. The condition can be resolved through two significant approaches including medication and therapy.

Keywords: Anosognosia; Mental Health; Medication; Illustrated; Manifestation

\section{Anosognosia: How to Treat the Lack of Insight in Mental Health}

\section{Description}

Provision of mental health services is a right entitled to the persons affected owing to the anguish they undergo [1]. Anosognosia is a psychological condition that exists in the form of an individual lacking insight regarding the adversity of a medical condition [2]. The patient is unable to adequately discern the seriousness of the medical issue, which hinders their ability to seek medical assistance. The patient, Anna W., has demonstrated various symptoms associated with Anosognosia. The aspect of the patient calling contacting an emergency ambulance and later denying of such act is a significant element that would influence the diagnosis of Anosognosia. Pointedly, a majority of patients experiencing this psychological condition are prone to poor judgment regarding their conduct [3]. Also, the case description of the patient indicates that they have been trying to avoid their medication by giving excuses for their refusal. Importantly, inclination towards neglect regarding one's health by refusing to take medication is a significant symptom for the diagnosis of Anosognosia [4]. Moreover, the patient's family history is defined by 


\section{Psychology \& Psychological Research International Journal}

numerous mental issue as the father and brother have been diagnosed with schizophrenia and bipolar disorder respectively. Besides, the patient is currently on medication related to bipolar. The culmination of mental disorders is an attribute that increases the vulnerability of an individual to Anosognosia since their ability to make an informed decision on issues related to their health is significantly low [5]. Therefore, the diagnosis of the patient's condition is based on factual data as illustrated in the description of her situation as she was admittedto the hospital.

\section{Five Roles of Potential Contribution to Defending the Publication of Case Reports Recognition and Description of Anosognosia}

The early detection of Anosognosia is essential in ensuring that appropriate medical countermeasures are provided to the patient [6]. Through the early detection, it allows the psychiatrist and mental health providers to initiate structured counter-initiatives. In the case of Anna W., the discovery of the ailment was carried promptly as the emergency medical personnel assessed the inherent circumstances concerning the patient, which influenced the determination that the patient was experiencing severe psychological issues. The element of the patient of having a history of refusing to take her medication is a significant indicator that they ought to be diagnosed with Anosognosia. Significantly, by engaging in a cross analysis on the underlying factors influencing the manifestation of the ailment; it empowers the medical personnel to discern the correlation between the symptoms depicted by the patient with Anosognosia.

\section{Recognition of Manifestations of the Condition}

The indication of Anosognosia is an element that is defined by the prevalence of various components to validate the identification of the ailment. The existence adverse mental health is a significant indicator that offers the mental health providers as an appropriate platform for ruling out that the client has Anosognosia [7]. In the case of Anna W., she has had several episodes of a bipolarrelated maniac as evident in her decision to engage in irrational actions based on paranoia. Therefore, the manifestation of similar aspects is an illustration that the patient is indeed illustrating symptoms associated with Anosognosia. Moreover, a majority of research carried out regarding Anosognosia demonstrate that nearly half of bipolar and over $60 \%$ of schizophrenia diagnosed individuals are bound to experience Anosognosia [8]. Therefore, based on similar conclusions, it is apparent that there are higher chances that the patient is likely to be experiencing Anosognosia.

\section{Elucidation of the Mechanisms of the Condition}

The effective identification of the Anosognosia is a somewhat necessary step in understanding the most appropriate approach that the mental health provider can employ in resolving this issue. The management of the condition presents a wide range of challenges regarding the effective alleviation of the state. The existence of a similar situation is influenced by a wide range of problems attributable to the mental condition of the patient [9]. The effective management of Ann W. condition would be faced by various challenges, which are primarily influenced by her history with mental problems. The existence of delusion affects the effective management of the Anosognosia as the patient is not mentally composed to understand the significance of taking the initiative to seek medical assistance [10]. She presented non-bizarre delusions beliefs of something occurring in her life which is not out of the realm of possibility. Therefore, due to the associated paranoid conduct exhibited by the patient, the prevalence of a challenge on the management of the patient's condition is bound to take precedence concerning the provision of medical attention. Notably, the incidence of a challenge would be primarily characterized by the need of ensuring that the patient abides with the set medical recommendation. Additionally, given the historical engagement between the patient and family members, the aspect of establishing a lasting relationship with the patient is another notable challenge.

\section{Detection of Adverse Side Effects and Benefits of Drugs (And Other Treatments)}

A certified psychiatrist and mental health therapist undertake the diagnosis of Anosognosia by engaging the patient on different issues related to the condition. The options that the provider can employ are relatively extensive to include undertakings that are disposed towards ensuring that they understand the extent of the medical condition. Given the dynamic nature of the patients with mental issue prone to Anosognosia, it implies that the treatment team will utilize a broadened approach. The issuance of the positive and negative syndrome scale (PANSS) questionnaire to the patient is one of the essential methods used, which is aimed at assessing the cognitive capacity of the patient on understanding the significance of undertaking the appropriate measures concerning their health welfare [11]. Based on the results of the questionnaires, the 


\section{Psychology \& Psychological Research International Journal}

therapist can discern the most suitable approach that can be employed in addressing the issue. Besides, the concept of utilizing a computer imaging, such as the computed tomography (CT) scan, is a valid methodology that can be used for diagnosis of Anosognosia among patients with stroke [12]. The scan would demonstrate the areas of the brain that have been affected to increase the ability of the medical practitioner to undertake the relevant counterinitiatives. Given the scope of the above alternatives that can be employed in diagnosis undertaking, providing Ann W. with questionnaires would be the most viable methodology. Treating Anosognosia is an attribute that ought to be undertaken with utmost prudence owing to the dynamic nature of effectiveness on the particular patient. The utilization of an integrated approach, both medication and therapeutic, should be influenced by a wide range of factors, such as the severity of Anosognosia symptoms exhibited by the patient [13]. Given similar dynamics, it is evident that the side effects experienced by the patient will be unique. The following is an analysis of the various available options that can be utilized along with the inherent advantages as well as the side effects the patient is bound to experience.

Involuntary treatment is a medication approach that is characterized by compelling the patient to adopt a medication regime attributable to their low cognition on their conduct [14]. Nevertheless, undertaking this initiative necessitates compliance with the legal requirements regarding the state of the mind of the patient. Importantly, this approach befits the case of Ann $\mathrm{W}$. as she has demonstrated adverse symptoms associated with neglect on taking precaution to enhance her health welfare. The side effects of this approach are dynamic depending on the medication the patient has been prescribed. The advantage of this approach is that it influences the realization of enhanced results on dealing with the various adversities associated with the ailment. Furthermore, engaging in structured therapy geared towards understanding the condition of the patient is another treatment methodology [15].

Diagnosis of a patient with anosognosia is based upon clinical evaluation by a psychiatrist. For example, Chao and Kawasaki recommended an approach, which has the acronym "LEAP." It involves the following approaches:

Listening to the patient

Empathizing with the patient

Agreeing with the patient

Partnering with the patient
Montero (2018) has included the acronym ON (open to resistant, navigate patient's decision). The " 0 " is psychological resistance is the phenomenon often encountered in clinical practice in which patients either directly or indirectly exhibits paradoxical opposing behaviors in presumably a clinically initiated push and pull of a change process. Taking into consideration personal construct psychology where people never know the world directly, but only via a set of "personal constructs" that they create. "N" is for the clinician that should understand resistant of the patient by taking into consideration the construct. The clinician navigation tool is a two psychosocial first during the initial hospitalization and second when the individual is stabilized and almost ready for discharge. It can help understand the patient's construct and navigate patient's decision making giving the person always full autonomy of their decision. This "LEAP-ON" approach helps to take the patient into confidence by the doctor so that that treatment can be initiated as soon as possible. Noticeably, this methodology is based on formal sessions with the patients to facilitate the development of approaches that the patient can embrace. The procedure is advantageous as it influences the realization of perceptive changes, which are imperative in attaining improved mental welfare.

\section{Medical Education and Audit}

The aspect of engaging in awareness creation not only educates the patient but also an appropriate platform for the realization of changes regarding the conduct of the affected persons. On the case of Ann W., the most appropriate methodology would be engaging in structured consultations on a weekly basis. Through these encounters, the objective would be to ensure that her rationality on issues related to her health is improved. The realization of a similar goal would enhance her recovery.

\section{References}

1. McCance-Katz E (2018) Changing focus: The right to treatment of serious mental issues. Presentation. N.p.

2. Davies M, McGill C, Davies A (2018) Anosognosia for motor impairments as adelusion: Anomalies of experience and belief evaluation, 1-24.

3. Bayne T, Cleeremans A, Wilken P (2014) The Oxford companion to consciousness. Oxford: Oxford University Press. 


\section{Psychology \& Psychological Research International Journal}

4. Steketee G, Frost R (2013) Treatment for Hoarding Disorder. Oxford University Press, USA.

5. Buchmann I, Jung R, Liepert J, Randerath J (2018) Assessing Anosognosia in Apraxia of Common ToolUse With the VATA-NAT. Frontiersin Human Neuroscience 12.

6. Brandeis G (2017) The art of misdiagnosis. Beacon Press.

7. Senturk G, Bilgic B, Arslan A, Bayram A, Hanagasi H, et al. (2016) Cognitive and anatomical correlates of Anosognosia in amnestic mild cognitive impairment and early-stage Alzheimer's disease. International Psychogeriatrics 29(02): 293-302.

8. Silva R, Mograbi D, Camelo E, Santana C, LandeiraFernandez J, et al. (2017) Clinical correlates of loss of insight in bipolar depression. Trends in Psychiatry and Psychotherapy 39(4): 264-269.

9. Verhülsdonk S, Lange Asschenfeldt C, Höft B, Schwender H, Supprian T, et al. (2017) Repressive coping does not contribute to Anosognosia in first diagnosis patients with Alzheimer disease. Alzheimer Disease \& Associated Disorders 31(3): 249-255.

10. Orfei M, Assogna F, Pellicano C, Pontieri F, Caltagirone $\mathrm{C}$, et al. (2018) Anosognosia for cognitive and behavioral symptoms in Parkinson's disease with mild dementia and mild cognitive impairment: Frequency and neuropsychological/neuropsychiatric correlates. Parkinsonism Relat Disord S13538020(18): 30203-30307.

11. Lysaker P, Pattison M, Leonhardt B, Phelps S, Vohs J (2018) Insight in schizophrenia spectrum disorders: relationship with behavior, mood and perceived quality of life, underlying causes and emerging treatments. World Psychiatry 17(1): 12-23.

12. Manasco H (2017) Introduction to neurogenic communication disorders. Jones \& Bartlett Publishers.

13. Williams D, Kaji A (2018) Clinical Decision Aids or Clinician Gestalt? Hard to Know Which Is Bette. Annals of Emergency Medicine 71(2): 256-261.

14. Giotakos 0 (2018) Poor insight in psychosis and meta-representation models. Dialoguesin Clinical Neuroscience \& Mental Health 1(1): 12-24.

15. Starkstein S (2014) Anosognosia in Alzheimer's disease: Diagnosis, frequency, mechanism and clinical correlates. Cortex 61: 64-73.

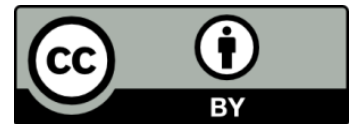

